\title{
Enhanced resolving power by moving field ion mobility spectrometry
}

\author{
Alexander Bohnhorst*, Moritz Hitzemann, Martin Lippmann, Ansgar T. Kirk, Stefan Zimmermann \\ Leibniz Universität Hannover, Institute of Electrical Engineering and Measurement Technology, Department of \\ Sensors and Measurement Technology, Appelstrasse 9A, 30167 Hannover, Germany \\ *Corresponding Author: Bohnhorst@geml.uni.hannover.de; Tel.: +49 5117623279.
}

\section{Table of Content}

1. Derivation of the MOF-IMS Model

2. MOF-IMS Control Logic 


\section{Derivation of the MOF-IMS Model}

Dependency of the ion mobility $(K)$, drift length $\left(L_{D}\right)$, drift time $\left(t_{D}\right)$ and drift voltage $\left(U_{D}\right)$.

$$
K=\frac{L_{D}^{2}}{t_{D} \cdot U_{D}}
$$

Definition of the resolving power $\left(R_{P}\right)$ as the ratio of drift time $\left(t_{D}\right)$ and full width at half maximum $\left(w_{0,5}\right)$.

$$
R_{P}=\frac{t_{D}}{w_{0,5}}
$$

Resolving power $\left(R_{P, o p t}\right)$ in the optimum operational point of the ion mobility spectrometer as a function of the optimum drift voltage $\left(U_{\text {opt }}\right)$ and the temperature $(T)$. Boltzmann constant $\left(k_{B}\right)$, elemental charge $(e)$ and all other constants can be approximated by a constant factor of 26.4 .

$$
R_{P, o p t}=\sqrt{\frac{e}{24 k_{B} \ln (2)}} \cdot \sqrt{\frac{U_{o p t}}{T}} \approx 26.4 \cdot \sqrt{\frac{U_{o p t}}{T}}
$$

Definition of the effective drift voltage $\left(U_{D, e f f}\right)$ in dependency of the number of segments in the drift region $\left(n_{\text {seg }}\right.$ ), the number of active segments during the transit $\left(n_{\text {active }}\right)$. The gain factor $(N)$ is defined as the ratio of $n_{\text {seg }}$ and $n_{\text {active }}$.

$$
U_{D, \text { eff }}=U_{D} \cdot \frac{n_{\text {seg }}}{n_{\text {active }}}=U_{D} \cdot N
$$

Insertion of Eq. 4 in Eq. 5 yield the resolving power $\left(R_{P, \text { mof }}\right)$ in MOF mode as a function of gain factor $N$ and the optimum resolving power $R_{P, \text { opt }}$ in static mode.

$$
\frac{R_{P, \text { mof }}}{R_{P, \text { opt }}}=\sqrt{\frac{n_{\text {seg }}}{n_{\text {active }}}}=\sqrt{N}
$$

In order to calculate the detectable mobility ratio in MOF mode we assume the difference in the distance covered by the fastest $\left(L_{\max }\right)$ and slowest ion $\left(L_{\min }\right)$ must be less than the length of the active segments $\left(L_{\text {active }}\right)$.

$$
L_{\text {active }}-w_{B} \leq L_{\max }-L_{\min }
$$

The length $L_{\text {active }}$ can be expressed by number $n_{\text {active }}$ and length $L_{\text {seg }}$ of the active segments. To avoid losses at the grids when switching the field, the base width $\left(w_{B}\right)$, which is the sum of the minimum distances between the centers of gravity of the ion distributions and the boundaries of the active area, is added to the active length. Finally, an active segment must always be switched over when switching the field. Since no ions may be in this segment at this time, $L_{\text {active }}$ must be reduced by $L_{\text {seg }}$.

$$
n_{\text {active }} \cdot L_{\text {seg }}-L_{\text {seg }}-w_{B} \leq L_{\max }-L_{\min }
$$


The distances $L_{\max }$ and $L_{\min }$ can be expressed with the maximum $\left(K_{\max }\right)$ and minimum mobility $\left(K_{\min }\right)$ according to Eq. 1Error! Reference source not found..

$$
n_{\text {active }} \cdot L_{\text {seg }}-L_{\text {seg }}-w_{B} \leq t \cdot K_{\max } \cdot E_{D, e f f}-t \cdot K_{\min } \cdot E_{D, \text { eff }}
$$

Reordering of Eq. 8 yield Eq. 9.

$$
\frac{n_{\text {active }} \cdot L_{s e g}-L_{s e g}-w_{B}}{t \cdot E_{D, \text { eff }}} \leq K_{\max }-K_{\min }
$$

The time $t$ is the time of the last switching operation. Since the fastest ion at this point in time is immediately before the transition to the last segment, $t$ can be expressed with the mobility $K_{\max }$ by Eq. 1 using the drift length until the last segment: $\left(n_{\text {seg }}-1\right) \cdot L_{\text {seg }}$.

$$
\frac{\left(n_{\text {active }} \cdot L_{s e g}-L_{s e g}-w_{B}\right) \cdot E_{D, \text { eff }} \cdot K_{\max }}{\left(n_{\mathrm{seg}}-1\right) \cdot L_{s e g} \cdot E_{D, \text { eff }}} \leq K_{\max }-K_{\min }
$$

Reordering of Eq. 10 yield Eq. 11.

$$
\frac{n_{\text {active }}-1-\frac{w_{B}}{L_{s e g}}}{n_{\text {seg }}-1} \leq 1-\frac{K_{\min }}{K_{\max }}
$$

Finally Eq. 11 can be used to calculate the ratio of the lowest and highest mobility detectable in MOF mode as a function of the gain factor $N$, the number of active segments $n_{\text {active, }}$ the length of each segment $L_{\text {seg }}$ and the base width of the peaks $w_{B}$.

$$
\frac{K_{\text {max }}}{K_{\text {min }}} \geq \frac{n_{\text {seg }}-1}{n_{\text {seg }}-n_{\text {active }}+\frac{w_{B}}{L_{\text {seg }}}}=\frac{n_{\text {active }} \cdot N-1}{(N-1) \cdot n_{\text {active }}+\frac{w_{B}}{L_{\text {seg }}}}
$$

The ratio of $w_{B}$ and $L_{s e g}$ indicates the space filling of a segment whereby $w_{B}$ can be expressed by the full width at half maximum $w_{0.5}$ and a conversion factor $\frac{4}{3.35}$. The drift velocity $v_{D}$ serves as a conversion factor between the temporal and spatial half-width. Changing and inserting Eq. 5 leads to Eq. 13.

$$
\frac{w_{B}}{L_{\text {seg }}}=\frac{\frac{4}{2.35} \cdot w_{0,5} \cdot v_{D}}{L_{\text {seg }}}=\frac{1.7 \cdot t_{D} \cdot v_{D}}{L_{\text {seg }} \cdot R_{P}}=\frac{1.7 \cdot L_{D}}{L_{\text {seg }} \cdot R_{P, \text { mof }}}=\frac{1.7 \cdot n_{\text {seg }}}{R_{P, \text { mof }}}=\frac{1.7 \cdot n_{\text {seg }}}{\sqrt{N} \cdot R_{P, \text { opt }}}=\frac{1.7 \cdot n_{\text {active }} \cdot \sqrt{N}}{R_{P, \text { opt }}}
$$

Inserting Eq. 13 into Eq. 12 ultimately provides the measurable mobility range.

$$
\frac{K_{\text {max }}}{K_{\min }}=\frac{N-\frac{1}{n_{\text {active }}}}{N+\sqrt{N} \frac{1.7}{R_{P, \text { opt }}}-1}
$$

To determine $N_{\text {crit }}$, Eq. 14 is rearranged. Furthermore, the space filling factor 1.7 must be doubled, to ensure that exactly two peaks will fit into the active region. Thus, by assuming a mobility ratio of 1 the gain factor $N$ yield $N_{\text {crit }}$. 


$$
\begin{gathered}
\frac{K_{\text {max }}}{K_{\text {min }}} \stackrel{\text { def }}{=} 1=\frac{N_{\text {crit }}-\frac{1}{n_{\text {active }}}}{N_{\text {crit }}+\sqrt{N_{\text {crit }} \frac{3.4}{R_{P, \text { opt }}}-1}} \\
N_{\text {crit }}+\sqrt{N_{\text {crit }}} \frac{3.4}{R_{P, \text { opt }}}-1=N_{\text {crit }}-\frac{1}{n_{\text {active }}}
\end{gathered}
$$

Rearranging yield the maximum possible gain factor Eq. 17 as a function of $R_{P, o p t}$ and $n_{\text {active }}$.

$$
N_{\text {crit }}=\left(1-\frac{1}{n_{\text {active }}}\right)^{2} \cdot \frac{R_{P, \text { opt }}^{2}}{3.4^{2}}
$$

Insertion of Eq. 17 into Eq. 5 offers the maximum possible resolving power $R_{P, \text { crit }}$ when utilizing a MOF-IMS with the gain factor $N_{\text {crit }}$.

$$
R_{P, \text { crit }}=\frac{R_{P, \text { opt }}^{2}}{3.4} \cdot\left(1-\frac{1}{n_{\text {active }}}\right)
$$

\section{MOF-IMS Control Logic}

The electric field in the MOF-IMS is controlled by the switches connected to each segment. In this setup n-channel MOS-FETs are used as switches. These can be opened and closed, electrically isolated by a digital control voltage. Transistors and isolators are integrated in a high-voltage switch manufactured by Behlke. As shown in Figure S1, a logic consisting of a 4-bit shift register and a series of OR and NOR gates is used to control the switch cascade. The logic ensures that at all times the two adjacent switches in the cascade are always open and all others are closed. The times at which the cascade switches to the next state can be calculated using the mobility of the ions, the drift voltage and the length of a segment according to Eq. 1.

The field in the lower part of Figure S1 shows in each row which switches are open (green) or closed (grey). Each row reflects a state the MOF-IMS can enter. Passing through all states corresponds to a complete switching sequence. Above the field with the MOF-IMS states the position of the ions is indicated, to which the next state should be taken. For example, the transition from the 1st to the 2nd state (transition from the first to the second row) should take place exactly when the ions are in the middle of the second segment - at a drift length of 37.5 $\mathrm{mm}$.

Eq. 1 can be used to convert the desired spatial position of the ions into temporal switching points. Since only two segments are active at a time, the voltage $U$ applied to the segments corresponds to half the drift voltage. In this case $1250 \mathrm{~V}$. For the mobility $\mathrm{K}$, for example, we use a mobility of $1.79 \mathrm{~cm}^{2} / \mathrm{Vs}$. This corresponds approximately to the mobility of the proton-bound dimer of acetone. Inserting provides us with the first switching point (Eq. 19). The switching points for all states calculated in this way are plotted below the field in the lower part of Figure S1.

$$
t_{\text {switch }}=\frac{L^{2}}{K \cdot U}=\frac{\left(37.5 \cdot 10^{-3} \mathrm{~m}\right)^{2}}{1.79 \cdot 10^{-4} \mathrm{~cm}^{2} / \mathrm{Vs} \cdot 1250 \mathrm{~V}}=6.28 \mathrm{~ms}
$$


To ensure that the drift region is free of ions which might remain in the segments when switching further, every second segment is supplied with voltage for a short time immediately after the spectrum. This discharges all remaining ions on the grids, preventing artifacts from forming in the spectrum.

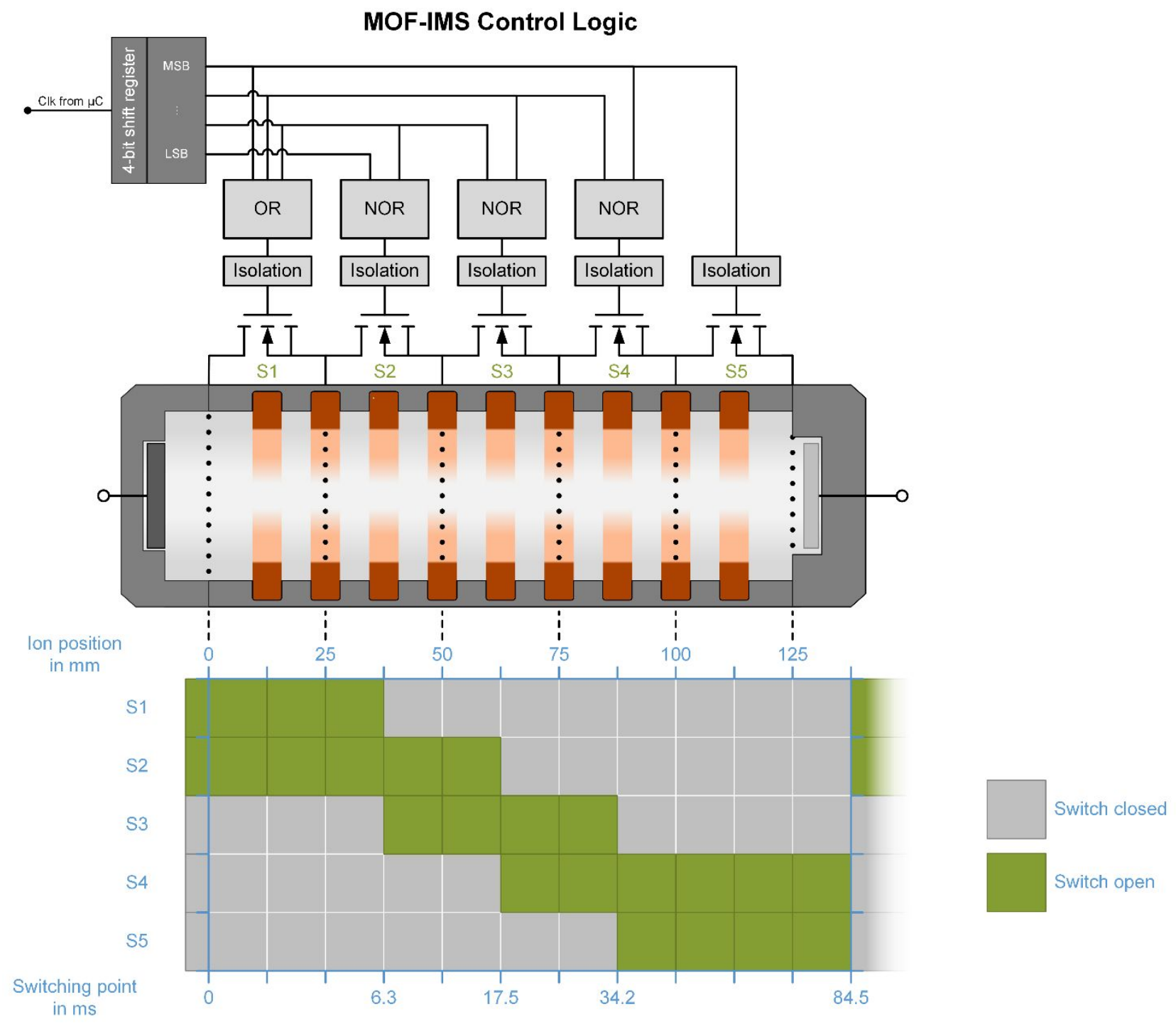

Figure S1: Diagram of the control logic of the MOF-IMS. The upper part of the figure shows the 4-bit shift register for the control of the MOS-FET switches. In the middle part a MOF-IMS with five segments is shown schematically. In the lower part of the figure the time sequence of the switching sequence is shown. During the green marked period, the switches are openthe respective segment is supplied with voltage. The switches should be switched in such a way that the ions are always exactly in the middle of a segment. The temporal switching points shown below are calculated from the length of the segments (here $25 \mathrm{~mm}$ ), the drift voltage (here $2.5 \mathrm{kV}$ ) and the average mobility of the ion species under consideration (here $1.79 \mathrm{~cm}^{2} / \mathrm{Vs}$ ). The 4-bit shift register is then switched to the desired points in time by means of a microcontroller. 